\title{
Artikel
}

Dennis Pausch*

\section{Ars invectiva und artifizielle Mündlichkeit: Schmähungen in Rom zwischen Schulbuch und scheinbarer Spontaneität}


Abstract: This paper starts from the question of how the perception of an invective in Late Republican Rome is influenced, when it follows strictly the rules of the ars rhetorica. Since speaker and audience will have undergone the same rhetorical training, both sides have clear ideas about the rules of the genre, as they can be reconstructed from the surviving textbooks. At the same time, it can be shown on the basis of ancient evidence that insults were perceived not only more effective, but also as socially more acceptable if they arose unprepared from the situation - or if they gave exactly this impression. In the case of invective, thus, a speaker must make a special effort not to let his preparation become visible. For this purpose, he can, on the one hand, resort to the technique of artificial orality and apparent spontaneity and, in this way, take the usual dissimulatio artis to extremes. On the other hand, he can deliberately deviate from the rules of textbooks, resulting in a strong tendency of invective to a permanent innovation. This will be demonstrated by the example of some passages from Cicero's speech pro Caelio from 56 BC.

Keywords: Rhetorik, Gattungsregeln, Erwartungen der Rezipienten, Innovation, artifizielle Mündlichkeit, simulierte Spontaneität, Cicero, Quintilian, Aristoteles - rhetoric, rules of genre, expectation of recipients, innovation, artificial orality, apparent spontaneity, Cicero, Quintilian, Aristotle

*Prof. Dr. Dennis Pausch, TU Dresden, Institut für Klassische Philologie, Professur für Klassische Philologie/Latein, dennis.pausch@tu-dresden.de

Schon die ersten Rhetoriklehrer im 5. Jh. v. Chr. dürften nicht nur versprochen haben, dass die Absolventen ihres Studienangebotes, die schwächere Seite zur stärkeren machen' können (Tòv

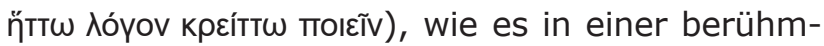
ten, zumeist auf Protagoras zurückgeführten Formulierung heißt, ${ }^{1}$ sondern doch wohl auch, dass sie in der Lage sein werden, mit den Mitteln der Sprache, den besseren Mann zum schlechteren zu machen'. Die Vermittlung der Fertigkeit zur verbalen Herabsetzung dürfte jedoch in der antiken Entsprechung der heute auf Hochglanzpapier gedruckten Werbebroschüren solcher Bildungsinstitute nicht allzu weit oben gestanden haben, zeigt sich die oft kritisierte moralische Ambiguität der

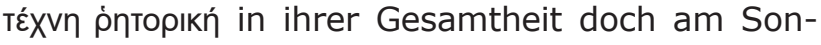
derfall der ars invectiva in besonderer Schärfe. ${ }^{2}$

1 Vgl. Aristoteles, Rhetorik 2,24 (1402a23) und Protagoras, Fragment 80 A 21.

2 Für einen gut lesbaren Überblick zur antiken Rhetorik und den mit ihr verbundenen Debatten vgl. Stroh (2009) Macht der Rede.
Die Autoren der rhetorischen Lehrwerke, die sich aus der Antike erhalten haben, reagieren auf diesen Vorwurf nicht zuletzt dadurch, dass sie die invektiven Kompetenzen nicht als solche und separat vermitteln, sondern immer in Verbindung mit der gegenläufigen Technik des Rühmens und Preisens. Es gibt also kein ,Modul Mobbing', um es in unseren Worten zu sagen, sondern ein Kapitel zu ,Loben und Tadeln' (ع̈naıvos / laus und чóyos / vituperatio), wobei immer von ersterem ausgegangen wird. Daraus ergibt sich dann die Möglichkeit, die Schmähung eher kurz zu behandeln, indem man am Ende festhält, dass dafür alle Empfehlungen nur umgekehrt befolgt werden müssen. ${ }^{3}$ Trotz der Zurückhaltung, die ,schwarze Kunst' der Invektive sozusagen ins Schaufenster zu stellen, spielen verbale Herabsetzungen nicht nur in den erhaltenen Reden eine große Rolle,

3 Vgl. z.B. Aristoteles, Rhetorik 1,9 (1368a35): ò yà yóyos

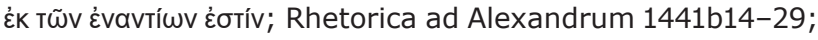
Rhetorica ad Herennium 3,10 und Quintilian, institutio oratoria 3,7,19; für weitere Stellen Adamietz (1966) de inventione und ad Herennium, S. 164, und Koster (1980) Invektive, S. 12-15. 
sondern sind auch in der rhetorischen Ausbildung intensiv behandelt worden.

Es ist aber weder das moralische Dilemma als solches noch der damit verbundene Unterschied zwischen Theorie und Praxis, die hier im Vordergrund stehen sollen, sondern das Problem der Erwartbarkeit, das sich ergibt, wenn beleidigende Äußerungen für den Redner, sein Gegenüber und sein Publikum nicht in erster Linie spontane und situative Reaktionen sind, sondern einen zentralen Teil der - wohl mehr oder weniger von allen drei Gruppen geteilten - Ausbildung und damit ihrer Vorannahmen an die Situation und die Textsorte bilden. Es handelt sich also - literaturwissenschaftlich gesprochen - um einen Sonderfall besonders starker und im Vorfeld vielfältig eingeübter Gattungsregeln. Die damit verbundene Vorhersehbarkeit steht jedoch im Widerspruch dazu, dass Schmähungen sonst zumeist stark auf die Situation bezogen sind, auf die sie in einer spontanen und oft impulsiven Weise reagieren oder zu reagieren vorgeben. ${ }^{4}$

\section{Die ars invectiva als Teil der rhetorischen Ausbildung in der Antike}

Die entsprechenden Erwartungen lassen sich sogar ziemlich präzise beschreiben, weil sich aus verschiedenen Epochen der Antike rhetorische Lehrwerke erhalten haben, die den Stoff nicht nur systematisch präsentieren und zum Teil mit Hilfe von Musterlösungen illustrieren, sondern sogar Hinweise auf die Art der Vermittlung enthalten. Die frühesten Beispiele stammen aus der Zeit des Hellenismus, wie die Rhetorik des Aristoteles ${ }^{5}$ und die sog. Rhetorica ad Alexandrum, die ebenfalls aus dem 4. Jh. v. Chr. stammt, heute aber nicht mehr als ein Werk des Aristoteles, sondern

\footnotetext{
4 Das Problem stellt sich für die Lobrede in ähnlicher, wenn auch nicht identischer Form: Zugespitzt könnte man sagen, dass auch eine gelungene laudatio immer ein gewisses $\mathrm{Maß}$ an Abweichung von der Konvention benötigt, weil die bloße Erfüllung der Erwartungen ansonsten selbst ein invektives Potential enthält (für diesen Hinweis aus der Diskussion weiß ich mich Marina Münkler sehr verbunden). 5 Vgl. allg. Rapp (2002) Aristoteles zum philosophischen Hintergrund und Piepenbrink (2020) Aristoteles zum historischen Kontext.
}

des Anaximenes von Lampsakos gilt. ${ }^{6}$ Eine gute Überlieferungssituation ergibt sich in Rom nicht zuletzt vom 1. Jh. v. Chr. bis zum 1. Jh. n. Chr., also etwa für die Zeit von Cicero bis Quintilian. Aber auch in der späteren Kaiserzeit und bis in die Spätantike sind weitere und für die Rezeption in Mittelalter und Neuzeit ebenfalls einflussreiche Werke entstanden. Dabei zeichnet sich eine Entwicklung ab, die uns nicht unvertraut vorkommen dürfte: Als Reaktion auf die offenbar nicht in allen Fällen gewährleistete Anschlussfähigkeit der Vorkenntnisse der angehenden Studenten etabliert sich das System der sog. Progymnasmata, der Vorübungen (es ist eigentlich ein Begriff aus dem Sport) ${ }^{7}$ durch deren vorgelagerter Absolvierung der Erfolg im Studium sichergestellt werden soll. Hier haben neben der Erzählung, dem Beweis oder der Ekphrasis auch die ,Trainingseinheiten' des Lobens und Tadelns ihren festen Platz. ${ }^{8}$

Im Laufe der Jahrhunderte ist natürlich noch mit weiteren Modifikationen zu rechnen, sodass Aussagen zur antiken Rhetorik in ihrer Gesamtheit stets nur mit Vorsicht zu treffen sind. Wenn wir jedoch im Rom der Späten Republik bleiben, lassen sich gleich aus zwei rhetorischen Schriften begründete Vermutungen über die Vorkenntnisse eines Redners und seiner Zuhörer gewinnen. So verweist Cicero in seiner Jugendschrift de inventione (ca. 85-80 v. Chr.), die er dem Auffinden von Ideen zur Behandlung des jeweiligen Themas gewidmet hat, für beide Arten von Argumenten, die sich ex personis gewinnen lassen, also sowohl für die positive wie für die negative Charakterisie-

6 Vgl. nach wie vor grundlegend Fuhrmann (1965) Alexander-Rhetorik sowie ferner Chiron (2011) Rhetoric to Alexander.

7 Zu den Progymnasmata vgl. jetzt allg. Berardi (2017) Esercizi preparatori.

8 So schon bei Quintilian am Ende des 1. Jh. n. Chr. (institutio oratoria 2,4,20). Die griechischen Progymnasmata-Handbücher aus der Spätantike (zusammengestellt bei Kennedy [2003] Progymnasmata und Patillon [2008-2014] Corpus rhetoricum) enthalten zum Teil Musterlösungen; so wird die Invektive bei Aphthonios von Antiochia (4. Jh. n. Chr.) mit einer fiktiven Rede gegen Philipp II. von Makedonien illustriert (Progymnasmata 9; vgl. für eine Übersetzung Schönberger [2019] Aphthonios). Solche Texte fehlen aus Rom, doch haben sich in der Appendix Sallustiana zwei Reden erhalten, die Sallust gegen Cicero und vice versa gehalten haben soll, die aber wohl einem Schulkontext entstammen (vgl. Koster [1980] Invektive, S. 177-200; Novokhatko [2009] Invectives, v.a. S. 3-18, und Keeline [2018] Reception of Cicero, S. 147-195, v.a. S. 147-151). 
rung auf dieselben Themenfelder. Bevor er diese im Folgenden weiter ausdifferenziert, ${ }^{9}$ fasst er sie in dieser Weise zusammen: "ac personis has res attributas putamus: nomen, naturam, victum, fortunam, habitum, affectionem, studia, consilia, facta, casus, orationes." (Und den Personen kommt, wie ich glaube, dies zu: Name, Natur, Lebensweise, Schicksal, persönliche Eigenschaft, Stimmung, Neigungen, Absichten, Taten, Zufälle, Reden. . ${ }^{10}$

Ganz ähnlich geht die in der gleichen Zeit entstandene und früher ebenfalls Cicero, heute aber einem anonymen Verfasser zugeschriebene Rhetorica ad Herennium vor, die noch stärker den Duktus eines Lehrbuchs aufweist. ${ }^{11}$ Der betreffende Abschnitt dort beginnt ebenfalls mit einer Matrix möglicher Themenfelder, an der sich der angehende Redner bei dem Arbeitsschritt der inventio, der Suche nach Argumenten, orientieren kann: ${ }^{12}$

Nunc ad demonstrativum genus causae transeamus. quoniam haec causa dividitur in laudem et vituperationem, quibus ex rebus laudem constituerimus, ex contrariis rebus erit vituperatio conparanda. laus igitur potest esse rerum externarum, corporis, amimi. rerum externarum sunt ea, quae casu aut fortuna secunda aut adversa accidere possunt: genus, educatio, divitiae, potestates, gloriae, civitas, amicitiae, et quae huiusmodi sunt et quae his contraria. corporis sunt ea, quae natura corpori adtribuit commoda aut incommoda: velocitas, vires, dignitas, valetudo et quae contraria sunt. animi sunt ea, quae consilio et cogitatione nostra constant: prudentia, iustitia, fortitudo, modestia et quae contraria sunt.

(Nun will ich zur darlegenden Redegattung übergehen. Da ja diese Rede in Lob und Tadel unterteilt ist, muß die tadelnde Rede aus den gegensätzlichen Punkten dazu gebildet werden, aus denen wir die Lobrede zusammenstellen. Das Lob kann also äußere Umstände, den Körper und den Geist betreffen. Zu den äußeren Umständen zählt man das, was durch Zufall oder Schicksal günstig oder ungünstig ausfallen kann: Herkunft, Erziehung, Reichtum, Macht, Ruhm, Bürgerrecht,

9 Vgl. Cicero, de inventione 1,34-36 sowie ferner 2,28-31 und 2,177-178 mit z.B. Koster (1980) Invektive, S. 17f., der zudem auf Ciceros Behandlung des Themas in seinen partitiones oratoriae verweist (§§70-82).

$10 \mathrm{Vgl}$. Cicero, de inventione 1,34, Übersetzung Nüßlein (1998).

11 Für einen eingehenden Vergleich beider Schriften vgl. Adamietz (1960) de inventione und ad Herennium.

12 Vgl. Rhetorica ad Herennium 3,10, Übersetzung Nüßlein (1994).
Freundschaften und dergleichen und die Nachteile, die im Gegensatz dazu stehen. Zu den körperlichen Eigenschaften zählt man die Vorteile und Nachteile, die die Natur dem Körper zugeteilt hat: Schnelligkeit, Kraft, würdevolles Auftreten, Gesundheit und die Gegensätze dazu. Zu den geistigen Eigenschaften zählt man, was auf unserer Überlegung und unserem Denken beruht: Klugheit, Gerechtigkeit, Tapferkeit, Selbstbeherrschung und die Gegensätze dazu.)

Anders als bei Cicero, der sich in seiner Schrift nur mit der Themenfindung beschäftigt, werden hier noch weitere Arbeitsschritte behandelt. So erhält der Redner im Folgenden zunächst einige Empfehlungen, welche Einleitung er wählen soll, um sein Publikum für das Lob oder den Tadel gewogen zu stimmen. ${ }^{13}$ Danach folgen konkrete Hinweise, wie er die nach dem obigen Raster gefundenen Ideen anordnen soll, was dem Arbeitsschritt der dispositio oder divisio entspricht: ${ }^{14}$

Divisione hac utemur: exponemus, quas res laudaturi sumus aut vituperaturi; deinde, ut quaeque, quove tempore res erit gesta, ordine dicemus, ut, quid quamque tute cauteque egerit, intellegatur. sed exponere oportebit animi virtutes aut vitia; deinde commoda aut incommoda corporis aut rerum externarum, quomodo ab animo tractata sint, demonstrare. ordinem hunc adhibere in demonstranda vita debemus: ab externis rebus: genus, in laude: quibus maioribus natus sit; si bono genere, parem aut excelsiorem fuisse; si humili genere, ipsum in suis, non in maiorum virtutibus habuisse praesidium; in vituperatione: si bono genere, dedecori maioribus fuisse; si malo, tamen his ipsis detrimento fuisse. educatio in laude: bene et honeste in bonis disciplinis per omnem pueritiam educatum; in vituperatione: inde se retraxisse aperte.

[14] a corporis commodis: natura si sit dignitas atque forma, laudi fuisse eam, non quemadmodum ceteris detrimento atque dedecori; si vires atque velocitas egregia, honestis haec exercitationibus et industriis dicemus conparata; si valetudo perpetua, diligentia et temperantia cupiditatum; in vituperatione, si erunt haec corporis commoda, male his usum dicemus, quae casu et natura tamquam quilibet gladiator habuerit; si non erunt, praeter formam omnia ipsius culpa et intemperantia afuisse dicemus. deinde <re>vertemur ad extraneas res, et in his animi virtutes aut vitia, quae fuerint, considerabimus; divitiae an paupertas fuerit, et quae potestates, quae gloriae, quae amici-

$13 \mathrm{Vgl}$. Rhetorica ad Herennium 3,11-12, Übersetzung Nüßlein (1994).

$14 \mathrm{Vgl}$. Rhetorica ad Herennium 3,13-15, Übersetzung Nüßlein (1994). 
tiae, quae inimicitiae, et quid fortiter inimicitiis gerundis fecerit; cuius causa susceperit inimicitias; qua fide, benivolentia, officio gesserit amicitias; in divitiis qualis aut paupertate cuiusmodi fuerit; quemadmodum habuerit in potestatibus gerundis animum. si interierit, cuiusmodi <mors eius fuerit, cuiusmodi> res mortem eius sit consecuta. [15] ad omnes autem res, in quibus animus hominis maxime consideratur, illae quattuor animi virtutes erunt adcommodandae; ut, si laudemus, aliud iuste, aliud fortiter, aliud modeste, aliud prudenter factum esse dicamus; si vituperabimus, <aliud iniuste, > aliud inmodeste, aliud ignave, aliud stulte factum praedicemus. perspicuum est iam nimirum ex hac dispositione, quemadmodum sit tractanda tripertita divisio laudis et vituperationis, si illud etiam adsumpserimus, non necesse esse nos omnes has partes in laudem aut in vituperationem transferre, propterea quod saepe <ne $>$ incidunt <quidem, saepe ita tenuiter incidunt, $>$ ut non sint necessariae dictu. quapropter eas partes, quae firmissimae videbuntur, legere oportebit. conclusionibus brevibus utemur, <enumeratione ad exitum causae; in ipsa> causa crebras et breves amplificationes interponemus per locos communis.

(Die Gliederung des Stoffes wenden wir folgendermaßen an: Wir stellen fest, welche Punkte wir loben oder tadeln werden; dann werden wir der Reihe nach sagen, wie und zu welchem Zeitpunkt sich jeder einzelne Vorgang abspielte, damit man erkennt, was und wie umsichtig und vorsichtig er es getan hat. Aber man wird seine charakterlichen Vorzüge oder Fehler vorstellen müssen; dann muß man darlegen, wie seine körperlichen Vor- oder Nachteile oder die Vor- oder Nachteile der äußeren Umstände von seinem Charakter beeinflußt wurden. Bei der Darlegung seines Lebensweges müssen wir die folgende Reihenfolge einhalten: Ausgehend von den äußeren Umständen erwähnen wir seine Herkunft - in einer Lobrede, von welchen Vorfahren er abstammt; ist er von guter Herkunft, sagen wir, er sei seinen Vorfahren ebenbürtig oder überrage sie; ist er von niederer Herkunft, sagen wir, er habe Schutz gefunden in seinen eigenen Vorzügen, nicht in denen der Vorfahren. In einer tadelnden Rede sagen wir, wenn er von guter Herkunft ist, er habe seinen Vorfahren Schande gemacht; ist er von schlechter Herkunft, sagen wir, sogar diesen Vorfahren habe er geschadet. Was die Erziehung angeht, so sagen wir in einer Lobrede, er sei gut und ehrenhaft, in guten Wissenszweigen die ganze Jugend hindurch erzogen worden. In einer tadelnden Rede sagen wir, er habe sich offenkundig davon zurückgezogen.

[14] Ausgehend von körperlichen Vorzügen sagen wir: Wenn er von Natur aus eine würdige Erscheinung und Schönheit besitzt, diese bringe inm Ehre, nicht wie den übrigen Schaden und Schande; besitzt er Kraft und herausragende Schnelligkeit, werden wir sagen, diese habe er durch ehrenhafte Übungen und Fleiß erworben; besitzt er beständige Gesundheit, durch Gewissenhaftigkeit und Beherrschung der Begierden. In einer tadelnden Rede werden wir sagen, wenn diese körperlichen Vorzüge vorhanden sind, er habe schlechten Gebrauch von diesen Vorzügen gemacht, die er zufällig und von Natur aus besessen habe wie jeder beliebige Gladiator; sind sie nicht vorhanden, werden wir sagen, außer Schönheit hätten ihm alle Vorzüge durch seine eigene Schuld und Unbeherrschtheit gefehlt. Dann kehren wir wieder zu den äußeren Umständen zurück und dabei werden die charakterlichen Vorzüge oder Fehler, die vorhanden sind, betrachten; ob Reichtum oder Armut vorhanden war, welche Machtstellung, welcher Ruhm, welche Freundschaft, welche Feindschaft, und was er Tapferes vollbracht habe, indem er Feindschaften ausfocht; um wessen willen er Feindschaften auf sich genommen habe; mit welcher Zuverlässigkeit wohlwollender Gesinnung und Pflichttreue er Freundschaften gepflegt habe; wie er sich im Reichtum beziehungsweise auf welche Weise er sich in Armut verhalten habe, welche Gesinnung er bei der Ausübung von Macht gehabt habe. Wenn er gestorben ist, von welcher Art sein Tod gewesen und welches Ereignis auf seinen Tod gefolgt sei.

[15] Zu allen Umständen aber, in denen die Gesinnung eines Menschen am meisten in Betracht gezogen wird, muß man jene erwähnten Charaktertugenden in Bezug setzen, in der Weise, daß wir, wenn wir loben, sagen, das eine sei gerecht, anderes tapfer, anderes beherrscht, anderes klug getan worden; wenn wir tadeln, werden wir erklären, das eine sei ungerecht, anderes unbeherrscht, anderes feige, anderes töricht getan worden. Klar ersichtlich ist schon ohne Zweifel aus dieser Anordnung, wie die dreigeteilte Gliederung des Stoffes in der Lobrede und tadelnden Rede durchzuführen ist, wenn wir dazu auch noch berücksichtigen, daß wir nicht nur notwendigerweise alle diese Gesichtspunkte auf eine Lobrede und tadelnde Rede übertragen müssen, deswegen weil sie oft nicht einmal zutreffen und oft nur so wenig zutreffen, daß es nicht notwendig ist, sie zu erwähnen. Deswegen muß man die Gesichtspunkte auswählen, die als die beweiskräftigsten erscheinen. Den Schluß halten wir kurz, wir verwenden eine Aufzählung am Ende der Rede, in die Rede selbst flechten wir häufige, kurze Steigerungen ein mit Hilfe von Gemeinplätzen.)

Am Ende des Abschnitts erfolgt dann noch der Hinweis, dass der angehende orator, auch wenn er möglicherweise nicht allzu oft eine reine vituperatio wird halten müssen, ${ }^{15}$ die Kompetenzen

15 Dass es im Gegensatz zur Lobrede nur wenige soziale Anlässe für eine Tadelrede per se gibt, betont zu Recht Powell (2007) Invectice, S. 4: "The epideictic use of epainos is quite clear: public and ceremonial occasions of many kinds call for it. What is the epideictic use of psogos? This is not 
des Lobens und des Tadelns dennoch bei vielen anderen Gelegenheiten brauchen wird und er deswegen nicht weniger Fleiß auf diesen Teil seiner Ausbildung verwenden soll: ${ }^{16}$

nec hoc genus causa eo, quod raro accidit in vita, neglegentius commendandum est: neque enim id, quod potest accidere, ut faciundum sit aliquando, non oportet velle quam adcommodatissme posse facere; et si separatim haec causa minus saepe tractatur, at in iudicalibus et in deliberativis causis saepe magnae partes versantur laudis aut vituperationis. quare in hoc quoque causae genere nonnihil industriae consumendum putavimus.

\begin{abstract}
(Aber man darf diese Redegattung, deswegen, weil sie im Leben selten vorkommt, nicht weniger sorgfältig anempfehlen; denn man muß sehr wohl den Wunsch haben, auch das, was man nur hie und da tun muß, möglichst angemessen zu tun; und wenn auch diese Rede für sich getrennt weniger oft gehalten wird, kommen doch in Gerichts- und beratenden Reden oft große Abschnitte von Lob und Tadel vor. Deshalb muß man, wie ich glaube, auch für diese Redegattung nicht unerheblichen Fleiß aufbringen.)
\end{abstract}

Das ist einerseits ein interessanter Beleg dafür, dass die Invektive schon in der Antike nicht nur als eine separate Subgattung der Rhetorik, sondern zugleich als ein zentraler modus dicendi, als invective mode, angesehen wurde, der integraler Bestandteil anderer Textsorten sein konnte. ${ }^{17}$

Andererseits kann die energische Aufforderung, sich mit den sprachlichen Techniken für Lob und Tadel im Rahmen seiner rhetorischen Ausbildung ausführlich zu beschäftigen, noch einmal verdeutlichen, dass man davon ausgehen kann, dass die meisten Angehörigen der römischen Oberschicht solche, recht detailliert und jedenfalls stark normativ formulierten Empfehlungen entweder aus ihrer eigenen Zeit beim Rhetorik-

so clear, and in fact we meet the pure genre of psogos largely if not exclusively as a form of rhetorical exercise. It seems to have been allocated to epideictic for no better reason than that the theorists, with their tidy minds, liked opposites to belong together in the same genus."

$16 \mathrm{Vgl}$. Rhetorica ad Herennium 3,15, Übersetzung Nüßlein (1994) mit z.B. Powell (2007) Invective, S. 4.

17 Das zeigen auch die Schwierigkeiten, auf die Quintilian bei der Einteilung der genera orationis trifft, da für inn - im Gegensatz zu Aristoteles - Lob und Tadel sich nicht nur auf die epideiktischen Reden beschränken, sondern Elemente jeder Art von Rede sein können (vgl. Quintilian, institutio oratoria 3,4,1-16; mit Adamietz [1966] Quintiliani liber III, S. 93-100). lehrer oder (seltener) aus der autodidaktischen Lektüre der einschlägigen Lehrwerke in- und auswendig kannten. Ein solches Vorwissen kann nicht ohne Folgen für die Erwartbarkeit und damit aber auch für die Wirkung von Schmähungen und Beleidigungen geblieben sein.

\section{Das Problem der Erwartbarkeit und das Simulieren von Spontaneität}

Der Verlust von Überzeugungskraft durch die Vorhersehbarkeit der Argumentation ist natürlich ein generelles Dilemma, das die Rhetorik in ihrer Gesamtheit betrifft. Wie ein Rezipient auf einen Vortrag lege artis reagiert, der dieselbe Schule durchlaufen hat, wird durchaus gelegentlich thematisiert, etwa von Cicero, wenn er im Vorfeld des Verres-Prozesses seinem Konkurrenten Caecilius, der sich gleichfalls für die Übernahme der Anklage beworben hat, vorwirft, dessen Redekunst ginge nicht über die Empfehlungen hinaus, die sein Rhetoriklehrer für inn zusammengestellt habe, und er sei unter anderem deswegen der Aufgabe nicht gewachsen. ${ }^{18}$ Noch klarer bringt Seneca der Jüngere das Problem auf den Punkt, wenn er in einem seiner Briefe an Lucilius, in dem er diesen von der Furcht vor einem bevorstehenden Prozess befreien will, sich selbst unterbricht und inn als fictus interlocutur folgenden Einwand vorbringen lässt: "', decantatae' inquis , in omnibus

18 Vgl. v.a. Cicero, in Caecilium 47: "si enim mihi hodie respondere ad haec quae dico potueris, si ab isto libro, quem tibi magister ludi nescio qui ex alienis orationibus compositum dedit, verbo uno discesseris, posse te et illi quoque iudicio non deesse et causae atque officio tuo satis facere arbitrabor; sin mecum in hac prolusione nihil fueris, quem te in ipsa pugna cum acerrimo adversario fore putemus?" (Solltest du mir nämlich heute auf das, was ich sage, antworten können, solltest du von den Aufzeichnungen, die dir wer weiß welcher Schulmeister aus den Reden anderer Leute zusammengestellt hat, auch nur mit einem Worte abweichen, dann will ich glauben, daß du auch in dem anderen Verfahren deinen Mann stehen sowie der Sache und deiner Pflicht gerecht werden kannst; solltest du aber schon in diesem Vorgeplänkel mit mir ein Nichts sein, was müssen wir annehmen, wirst du erst sein, wenn du zur eigentlichen Schlacht mit dem grimmigen Gegner antrittst? - Übersetzung Fuhrmann [1995]). Vgl. ferner Cicero, in Caecilium 52. 
scholis fabulae istae sunt; iam mihi, cum ad contemnendam mortem ventum fuerit, Catonem narrabis." ("Abgedroschene Geschichten sind das", wirst Du sagen, "die in allen Schulen heruntergeleiert werden; gleich wirst Du mir, wenn es um die Todesverachtung geht, etwas von Cato erzählen."). ${ }^{19}$ Gemeint ist Cato der Jüngere und sein berühmter Selbstmord in Utica 46 v. Chr. mit dem Ziel, als freier Römer nicht unter der Diktatur Caesars leben zu müssen. Auch wenn es sich hier um ein exemplum und daher um einen Sonderfall handelt, ${ }^{20}$ illustriert die Stelle doch gut die Einbußen an Originalität und damit an Überzeugungskraft, die mit der Erwartbarkeit von Inhalt und Aufbau einhergehen. ${ }^{21}$

Für dieses prinzipielle Dilemma, dass auch eine wohl präparierte Rede weniger überzeugend wirkt, sobald man ihr eben diese Vorbereitung anmerkt, hat die antike Rhetorik die Empfehlung entwickelt, dass zu den Qualitäten eines wahrhaft guten Redners gerade auch die dissimulatio artis gehört, also die Fähigkeit, die eigene Kunstfertigkeit vor den Zuhörern zu verbergen. ${ }^{22}$ Ovid hat diesen Gedanken allgemein auf jede künstleri-

19 Seneca, epistulae morales 24,6, eigene Übersetzung. 20 Zur Bedeutung der exempla für die römische Kultur vgl. jetzt allg. Langlands (2018) Exemplary ethics und Roller (2018) Models from the past.

21 Vgl. Craig (2004) Audience Expectations, der am Beispiel von Ciceros Rede pro Milone der Frage nachgeht, wie glaubwürdig ,kunstgemäße' Anschuldigungen sind. Interessant ist unter anderem die Beobachtung, dass auch die ausbleibende oder nur flüchtige Behandlung eines erwarteten Punktes von der Gegenseite als Argument für ihre Sache verwendet werden kann (vgl. Craig [2004] Audience Expectations, S. 193f., mit Verweis auf Cicero, pro Fonteio 37-40 und pro Murena 11-14).

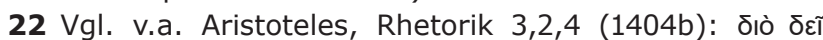

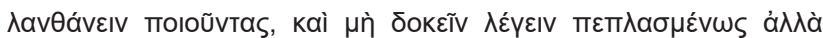

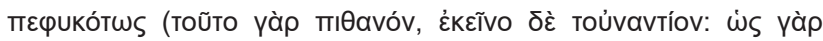

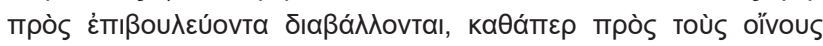

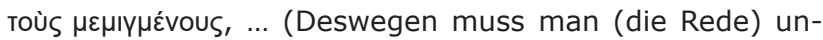
merklich komponieren und nicht den Anschein des gekünstelten, sondern des natürlichen Redens erwecken - diese nämlich ist überzeugend, jenes aber das Gegenteil, denn (die Zuhörer) lehnen es ab, wie gegenüber jemanden, der etwas im Schilde führt, wie bei den gemischten Weinen [...]. Übersetzung Rapp (2002). Vgl. ferner Piepenbrink (2020) Aristoteles, S. 151-156; Alkidamas, Über die Verfasser schriftlicher Reden oder über die Sophisten, 12-13; Rhetorica ad Herennium 4,10; Cicero, de oratore 2,153; Ps.-Longinus, Peri Hypsous 17-18; Quintilian, institutio oratoria 4,1,9; 11,1,15-16;12,9,5, sowie allg. z.B. Andersen (1996) Lingua suspecta; Hesk (1999) Rhetoric of Anti- sche Hervorbringung bezogen und im Kontext der Pygmalion-Geschichte in den Metamorphosen in der paradoxen Formulierung "ars adeo latet arte sua" auf den Punkt gebracht und so zum geflügelten Wort werden lassen. ${ }^{23}$

Im Falle invektiver Texte kommt aber noch eine weitere Ebene hinzu: Vorbereitete und nach allen Regeln der Kunst durchgestaltete Beleidigungen werden als schwerwiegender empfunden als spontane und daher nicht zuletzt als emotionaler Kontrollverlust erklärbare Ausfälle. Dass diese allgemeine Beobachtung auch für die Antike gilt, zeigt eine Stelle in den Tischgesprächen Plutarchs (ca. 45-125 n. Chr.) in wünschenswerter Deutlichkeit. Ein Kapitel dieses Werkes ist der Frage gewidmet, welche Scherze im Rahmen eines Symposions statthaft sind und welche nicht. ${ }^{24} \mathrm{Am}$ Ende dieses Abschnitts findet sich dann die folgende Bemerkung: ${ }^{25}$

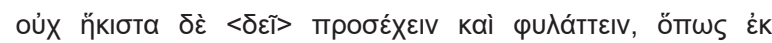

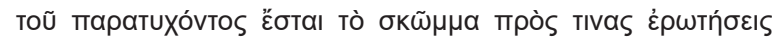

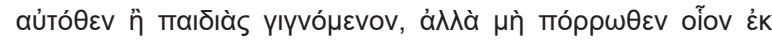

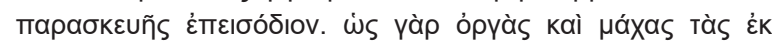

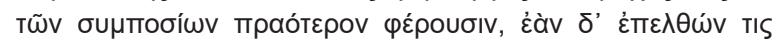

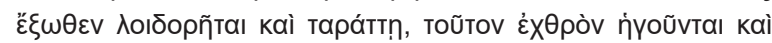

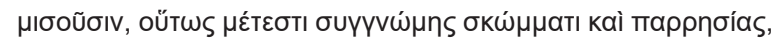

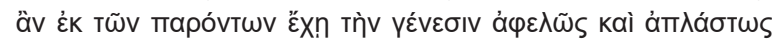

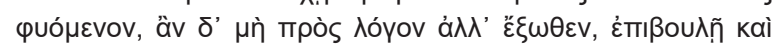

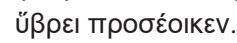

Nicht zuletzt muss man aber darauf schauen und achten, dass eine spöttische Bemerkung unmittelbar und ohne weiteres auf die Fragen oder Scherze eines anderen erfolgt, jedoch nicht als von langem vorbereitet oder als einstudierte Erwiderung erscheint. Denn auf Zank und Streitigkeiten, die aus dem Gelage heraus entstehen, reagieren wir vergleichsweise nachsichtig, wenn aber jemand von außen dazukommt und lästert und Unruhe stiftet, halten ihn alle für einen Feind und hassen ihn. Ebenso wird einer spöttischen Bemerkung oder einer freimütigen Äußerung Nachsicht zuteil, wenn ihr Ursprung im Augenblick liegt und sie ohne Vorbereitung und Hintergedanken entstanden ist; wenn sie aber nicht zur Sache gehört, sondern von außen dazukommt, wird sie als Absicht und Kränkung wahrgenommen.

rhetoric; Till (2009) Verbergen der Kunst, und Schloemann (2019) Kunst der freien Rede, v.a. S. 37-77.

23 Ovid, Metamorphosen 10,253.

24 Plutarch, quaestiones convivales 2,1,13.

25 Plutarch, quaestiones convivales 2,1,13 (634d-e), eigene Übersetzung. 
Plutarch argumentiert hier vor allem defensiv und betont, dass spontane Schmähungen weniger anstößig sind. Sie sind zugleich natürlicher aber auch treffender und effizienter, wie wiederum Cicero festhält, dessen rhetorisches Hauptwerk, der 55 v. Chr. entstandene Dialog de oratore, im zweiten Buch einen Exkurs zum Humor und zur richtigen Verwendung des Witzes enthält. ${ }^{26}$ Hier legt er Marcus Antonius, einem der Hauptunterredner, die folgenden Worte in den Mund: ${ }^{27}$

\begin{abstract}
omnino probabiliora sunt, quae lacessiti dicimus, quam quae priores; nam et ingenii celeritas maior est, quae apparet in respondendo, et humanitatis est responsio. videmur enim quieturi fuisse, nisi essemus lacessiti; $[\ldots]$.
\end{abstract}

Überhaupt überzeugt mehr, was wir auf eine Herausforderung hin sagen, als was wir vorher vorbringen; denn größer ist die Schlagfertigkeit und geistige Regsamkeit, welche beim Entgegnen in Erscheinung tritt, und es ist zudem menschlich, mit gleicher Münze heimzuzahlen. Wir erwecken nämlich den Eindruck, dass wir ruhig geblieben wären, wenn man uns nicht gereizt hätte.

Das von Plutarch für den gesellschaftlichen Kontext des Symposions und von Cicero zunächst einmal für die mündliche performance des Redners beschriebene Phänomen lässt sich auch auf schriftlich fixierte Beleidigungen übertragen, obwohl in diesem Fall seitens des Rezipienten natürlich immer das Wissen um eine Vorbereitung durch den Verfasser vorausgesetzt werden kann. Dennoch scheinen viele invektive Texte mit erheblichem sprachlichem Aufwand die Illusion erzeugen zu wollen, dass es sich bei ihnen um spontane Reaktionen auf reale Situationen handelt. Zum einschlägigen Repertoire gehören fingierte Apostrophen ebenso wie Dialoge mit dem Gegner oder Publikum, in denen vermeintliche Reaktionen eingeblendet werden, und viele andere sprachliche Strategien, die der Erzeugung artifizieller Mündlichkeit dienen.

Solche Elemente finden sich in poetischen Texten wie der jambischen Lyrik, der Satire oder

26 Vgl. Cicero, de oratore 2,216-290 mit Fantham (2004) Roman World, S. 186-208, und Beard (2014) Laughter in ancient Rome, S. 99-128.

$27 \mathrm{Vgl}$. Cicero, de oratore 2,230, Übersetzung Nüßlein (2007), sowie 2,246 (Witze rufen weniger Lachen hervor, wenn sie vorbereitet wirken: „ea, quia meditata putantur esse, minus ridentur"). dem Epigramm ebenso in vielen der Reden und zwar gerade auch in denjenigen, von denen wir wissen, dass sie entweder gar nicht gehalten oder vor der Publikation stark überarbeitet wurden. Aufgrund der einseitigen Überlieferungssituation stammen so gut wie alle erhaltenen Beispiele für die rednerische Praxis in Rom von Cicero, der in der Regel zudem als Herausgeber fungiert hat. Es ist daher wenig überraschend, dass die Frage, ob und wie stark sich seine publizierten Reden von den tatsächlich gehaltenen unterscheiden, bereits auf eine lange Forschungstradition zurückblicken kann. ${ }^{28}$ In diesem Kontext hat aber bislang der Sonderfall invektiver Reden mit inrem gesteigerten Interesse daran, reale Situationen als Entstehungskontext zu reklamieren und für den Leser präsent zu halten, noch keine angemessene Berücksichtigung gefunden.

Das Paradebeispiel hierfür stellt die actio secunda von Ciceros Reden gegen Verres dar. Durch den freiwilligen Gang des Angeklagten ins Exil nach Massilia hat die eigentlich vorgesehene zweite Verhandlung nicht mehr stattgefunden, was Cicero aber nicht davon abgehalten hat, seine Ausführungen während des fiktiven weiteren Prozessverlaufs gleichwohl in die Publikation der Reden eingehen zu lassen. Tatsächlich bildet diese actio secunda sogar den weitaus größeren Teil dieses Gesamtwerkes. Die hier in großer Zahl zutage tretenden Strategien zur Erzeugung artifizieller Mündlichkeit und scheinbarer Spontaneität sind daher sicherlich auch ein Element des literarischen Spiels und sollen zur Plausibilisierung der Fiktion einer weiteren Verhandlung beitragen, sie dienen zugleich aber sowohl zur Legitimation der verbalen Aggression als Teil des emotionalen Prozessgeschehens als auch zur Steigerung ihrer Wirkung, weil sich viele der Beleidigungen gegen Verres gleichsam aus der Situation zu ergeben scheinen.

Während also die rhetorischen Lehrbücher jedenfalls soweit sich diese aus der Antike erhalten haben - zwar allgemeine Empfehlungen zur dissimulatio artis enthalten, aber keine konkreten Hinweise geben, wie sich das Doppelproblem der erhöhten Legitimation und der verminderten Wirkung vorbereiteter Schmähungen umgehen lässt,

28 Vgl. v.a. Humbert (1925) Plaidoyers; Stroh (1975) Taxis und Taktik, S. 31-54; Blänsdorf (2001) Mündlichkeit; Steel (2013) Cicero; La Bua (2019) Cicero and Roman education, S. 33-42. 
lassen sich Ciceros publizierte Invektiven als best practice-Beispiele unter anderem genau hierfür verstehen. Da gerade die Verrinen, aber auch die Rede gegen Piso offenbar recht schnell und schon von den Zeitgenossen als Schultexte verwendet wurden, ${ }^{29}$ kann man berechtigterweise vermuten, dass bei ihrer Behandlung gerade auch diese Aspekte das Interesse der angehenden Redner gefunden haben werden.

\section{Ciceros Rede pro Caelio und die Beleidigungen als Improvisationstheater}

Anstelle dieser besonders naheliegenden Beispiele soll hier mit der oratio pro Caelio auf eine Verteidigungsrede aus Ciceros umfangreicher Tätigkeit als Anwalt näher eingegangen werden, in der man invektive Elemente auf den ersten Blick nicht vermuten würde, die aber tatsächlich ein besonders gutes Beispiel dafür liefert, wie weit die Redner in der Praxis gegangen sind, um das Problem von erwartbaren, nach allen Regeln der Schulbücher erfolgenden Beleidigungen und Schmähungen zu umgehen. Natürlich ist auch in diesem Fall mit Unterschieden zwischen seinem Agieren in der realen Situation des Prozesses und der späteren Publikation der Rede zu rechnen. Da wir in diesem Falle über keinerlei explizite Zeugnisse verfügen, ist es sogar umstritten, ob sie von Cicero selbst oder postum aus seinem Nachlass herausgegeben wurde. ${ }^{30}$

In beiden Fällen kann man jedoch davon ausgehen, dass die schriftliche Fassung nicht zuletzt eine bestimmte Imagination der konkreten Situation während der Verhandlung erzeugt, die mit

29 Vgl. z.B. Tempest (2013) Aspects of Performance, S. 70: "[...] the Verrines rapidly became popular as a model of prosecution oratory: an extract of the In Verrem (Verr. II.2.3-4) is among the earliest Latin papyri to be found in Egypt.", sowie für die Rede gegen Piso vgl. Cicero, epist. ad Quintum fratrem 3,1,11: "cum [...] meam (sc. orationem) in illum pueri omnes tamquam dictata perdiscant." (da meine Rede gegen jenen alle Knaben gleichsam als Musterlösung auswendig lernen.) mit z.B. Arena (2007), S. 152, und La Bua (2019) Cicero and Roman education, S. $25 \mathrm{f}$.

30 Für eine Übersicht über die Zeugnislage und die Forschungsdiskussion vgl. Dyck (2013) Pro Caelio, S. 25-28. den Intentionen des Redners beziehungsweise Autors weitgehend identisch sein dürfte.

Im Jahre 56 v. Chr. wurde M. Caelius Rufus unter anderem wegen Gewaltanwendung (nach der lex Plautia de vi) und versuchten Giftmords angeklagt. ${ }^{31}$ Der vermutlich etwa 30 -jährige Caelius war offenbar ein recht aktives Mitglied von Roms jeunesse dorée und möglicherweise auch einige Zeit der Liebhaber Clodias gewesen, der Schwester von Ciceros politischem Intimfeind Clodius Pulcher, die nach dem frühen Tod ihres Mannes Q. Caecilius Metellus Celer im Jahre 59 v. Chr. dank ihres finanziellen und familiären Hintergrundes ein für antike Verhältnisse ungewöhnlich selbstbestimmtes Leben geführt zu haben scheint. ${ }^{32}$ Jedenfalls will Cicero es so darstellen, der in dieser Rede ohnehin alles daransetzt, die beiden prominenten Geschwister als die treibenden Kräfte hinter der Anklage erscheinen zu lassen, die tatsächlich aber von dem erst 17-jährigen Sempronius Atratinus eingereicht wurde. Das soll dazu beitragen, die Aufmerksamkeit von den inhaltlichen Vorwürfen abzulenken und die Anklage stattdessen in moralischen Kategorien zu diskreditieren, indem er sie als einen durch Eifersucht motivierten Racheakt Clodias darstellt. ${ }^{33}$ Ihre Diffamierung bildet für Cicero somit ein zentrales Anliegen, das er unter anderem verfolgt, indem er ihr ein inzestuöses Verhältnis mit ihrem Bruder ${ }^{34}$ sowie die Ermordung ihres Gatten unterstellt. ${ }^{35}$ Der invektive Charakter der einschlägigen Abschnitte ist daher gerade in dieser Rede beson-

31 Da sich die Reden der Kläger nicht erhalten haben, lassen sich die Vorwürfe nicht mehr in allen Einzelheiten rekonstruieren; für eine Übersicht vgl. Dyck (2013) Pro Caelio, S. 2-8.

32 Zur Wahrnehmung Clodias durch die Zeitgenossen vgl. Günther (2000) Sexuelle Diffamierung; Hartmann (2007) Frauen in der Antike, S. 152-154, und Skinner (2011) Clodia Metelli. Zur vielverhandelten Frage, ob Clodia hinter Catulls Lesbia-Figur steht, z.B. Skinner (2011) Clodia Metelli, S. 121-145.

33 Es spricht einiges dafür, dass das Verhältnis der beiden im Ganzen eine Erfindung Ciceros ist (vgl. v.a. Stroh [1975] Taxis und Taktik, S. 243-298), beweisen lässt sich diese Annahme nach 2000 Jahren aber so wenig wie ihr Gegenteil.

34 Vgl. Cicero, pro Caelio 32; 36 und 78 mit Dyck (2013) Pro Caelio, S. 111, aber auch die wiederholten Andeutungen in seinen Briefen an Atticus $(2,9,1 ; 2,10,2 ; 2,14,1 ; 2,22,5$; $2,23,3)$ mit Harders (2008) Suavissima Soror, S. 237f., und Thurn (2018) Rufmord, S. $146 f$.

$35 \mathrm{Vgl}$. Cicero, pro Caelio 59-60. 
ders ausgeprägt, ${ }^{36}$ doch der Versuch, Gegnern oder Zeugen charakterliche Defizite zu unterstellen, spielt in der antiken Gerichtspraxis generell eine große Rolle und führt zu dem irritierenden Befund, dass Argumenten ad hominem oft mehr Bedeutung zukommt als den eigentlichen Sachgründen. ${ }^{37}$

Der hochgradig verletzende Charakter dieser Rede wird dadurch noch gesteigert, zugleich aber auch ein Stück weit gerechtfertigt und eingehegt, dass dieses an sich prosaische Plädoyer eines Verteidigers vor Gericht gleich auf mehreren Ebenen enge Bezüge zur Komödie aufweist. ${ }^{38}$ Damit steigert sich in diesem Fall noch einmal deutlich der theatralische Charakter, der ohnehin jeder Gerichtsverhandlung inhärent ist und der für die Antike in den letzten Jahren auch intensiv untersucht worden ist. ${ }^{39}$ Hierfür gibt es zunächst einen äußerlichen Grund: Die Verhandlung war unüblicherweise auch für den 4. April 56 v. Chr. angesetzt worden, an dem das Fest der Magna Mater begangen wurde, sodass zur gleichen Zeit und in räumlicher Nähe zu Ciceros Aufritt auf dem Forum ein umfangreiches Unterhaltungsprogramm geboten wurde, das nicht zuletzt Theateraufführungen umfasste. ${ }^{40}$ Vor diesem Hintergrund scheint er die Geschworenen und Zuhörer für ihr Kommen gleichsam entschädigen zu wollen, indem er sein Plädoyer zu einem unterhaltsamen ,Bühnenstück' macht und unter anderem viele Zitate aus dramatischen Texten einfließen lässt. ${ }^{41}$ Zugleich erweist sich dieses scheinbar nur aus der Okkasion geborene Entgegenkommen, wie die Forschung bereits überzeugend gezeigt hat, aber als zentraler Teil der Diffamierung der Anklage, die er im Gewand einer Komödienhandlung präsentiert, in der vor allem für Clodia

36 Vgl. v.a. Cicero, pro Caelio 30-36; 48-50; 57 und 62. 37 Vgl. allg. May (1988) Trials of Character, v.a. S. 1-12, und jetzt Thurn (2018) Rufmord.

$38 \mathrm{Zu}$ dieser Funktion der Anleihen an die Komödie in pro Caelio vgl. auch Pausch (im Erscheinen) Comic Invective.

39 Vgl. Bablitz (2007) Actors and Audience (zu Rom); Hall (2014) Judicial Theater (zu Cicero), und Papaioannou/ Serafim/da Vela (2017) Theatre of Justice (zur Antike).

40 Vgl. Cicero, pro Caelio 1-2 mit Geffken (1973) Comedy, S. 11-14, Volpe (1977) Humor, S. 313-316, und Leigh (2004) Comedy, S. 301-303.

41 Vgl. v.a. Cicero, pro Caelio 18; 36; 37 und 38, sowie ferner Zillinger (1911) Dichter, S. 64-68. nur die Rolle der moralisch fragwürdigen Hetäre (meretrix) bleibt. ${ }^{42}$

Die Anleihen an das Drama erweisen sich darüber hinaus als eine von mehreren Strategien, mit denen Cicero die Erwartbarkeit der Angriffe auf seine Gegner kaschieren und diese somit einerseits rechtfertigen, andererseits in ihrer Wirkung steigern möchte. ${ }^{43}$ Gezeigt werden soll das nun an einigen ausgewählten Passagen aus dem besonders invektiven Teil der Rede, in dem er sich Clodia als der vermeintlichen Hauptgegnerin seines Mandaten zuwendet, ${ }^{44}$ und an denen sich gleich mehrere der einschlägigen Techniken beobachten lassen: 45

sin ista muliere remota nec crimen ullum nec opes ad oppugnandum Caelium illis relinquuntur, quid est aliud quod nos patroni facere debeamus, nisi ut eos, qui insectantur, repellamus? quod quidem facerem vehementius, nisi intercederent mihi inimicitiae cum istius mulieris viro - fratre volui dicere; semper hic erro.

(Wenn aber diese Frau da unschädlich gemacht ist und ihnen weder irgendein Vorwurf noch eine Möglichkeit der Einflußnahme übriggelassen wird, um Caelius anzugreifen, was sollte ich als Verteidiger anderes tun, als diejenigen in ihrer Klage zurückzuweisen, die uns zusetzen? Dies würde ich gewiß energischer machen, wenn mir dabei nicht die Feindschaft zu ihrem Mann dazwischen käme - Bruder wollte ich sagen, da vertue ich mich jedesmal.)

Bereits der Verweis auf Clodia mit dem ebenso deiktischen wie despektierlichen Pronomen ista diese da - stellt einen Affront gegenüber einer Dame der römischen Oberschicht dar, den man sich in der konkreten Situation sicherlich noch durch das Zeigen mit dem Finger oder eine noch drastischere Geste verstärkt vorzustellen hat.

42 Vgl. v.a. Geffken (1973) Comedy; Arcellaschi (1997) Théâtre; Klodt (2003) Prozessparteien, S. 82-97, und Leigh (2004) Comedy sowie zu anderen Aspekten der Rede Austin (1960) Pro Caelio; Stroh (1975) Taxis und Taktik, S. 243-295; May (1988) Trials of Character, S. 105-116; Riggsby (1999) Crime and Community, S. 97-105, und Dyck (2013) Pro Caelio.

43 Vgl. aber auch Tatum (2007) Invective Identities, der die These vertritt, dass die Anleihen bei der Komödie Cicero vor allem dazu dienen, die Invektiven der anderen als konventionell, seine hingegen als besonders glaubwürdig darzustellen.

44 Vgl. Cicero, pro Caelio 30-36.

45 Vgl. Cicero, pro Caelio 32, Übersetzung Fuhrmann (1993). 
Ciceros vorgebliche Rücksichtnahme auf ihren Bruder Clodius, mit dem er - wie allgemein bekannt war - tatsächlich aber verfeindet war, dient dann natürlich nur dazu, den Geschwistern ein intimes Verhältnis zu unterstellen.

Inzest war als Teilbereich sexueller Devianz zwar ein Standardvorwurf in Theorie und Praxis, ${ }^{46}$ dennoch gibt sich Cicero hier Mühe, die Anschuldigung als einen Versprecher zu inszenieren. Dass es sich dabei tatsächlich um ein Versehen gehandelt habe, wird bereits durch die Übernahme in die publizierte Fassung der Rede überaus zweifelhaft. Gerade die leichte Durchschaubarkeit lenkt den Blick auf die Vorteile, die sich für den Redner aus der gespielten Spontaneität ergeben: Die Diffamierung Clodias wird dadurch- wenn natürlich auch nur scheinbar - entschuldigt, sie wird aber zugleich in ihrer Wirkung gesteigert, da sie sich nun mit dem Lachen über den inszenierten Lapsus verbindet und damit verstärkt. ${ }^{47}$

Die Technik eines absichtlichen Versprechers war unter dem Schlagwort der correctio oder der reprehensio im Übrigen ihrerseits Teil der rhetorischen Ausbildung, ${ }^{48}$ doch hier handelt es sich bei der Simulierung von Spontaneität in dieser Rede nicht um ein isoliertes Stilmittel, sondern um eine großflächigere Textstrategie, wie sich im Weiteren zeigt: ${ }^{49}$

nunc agam modice nec longius progrediar quam me mea fides et causa ipsa coget. neque enim muliebres umquam inimicitias mihi gerendas putavi, praesertim cum ea quam omnes semper amicam omnium potius quam cuiusquam inimicam putaverunt. [33] sed tamen ex ipsa quaeram prius utrum me secum severe et graviter et prisce agere malit an remisse et leniter et urbane.

46 Vgl. allg. Hickson-Hahn (1998) Laughter and Incest. 47 Vgl. Williams (2007) Pro Caelio, S. 123f. und Dyck (2013) Pro Caelio, S. 111 sowie zur Präsentation von Inzestvorwürfen durch Cicero Hickson-Hahn (1998) Laughter and Incest, S. 19-25, v.a. S. 20: "When the accusation of incest is couched in witty language, listeners may focus on the humorous technique rather than on the taboo content and the aggressive hostility. Moreover, there is always the 'only a joke' excuse. Listeners take pleasure in the penaltyfree expression of hostility and sexual aggression against the target and are won over to the side of the speaker who has given them that pleasure."

$48 \mathrm{Vgl}$. z.B. Rhetorica ad Herennium 4,36 sowie ferner Lausberg (1960) Handbuch, §§ 784-786.

49 Vgl. Cicero, pro Caelio 32-33, Übersetzung Fuhrmann (1993).
(Nun will ich besonnen vorgehen und nicht weiter ausholen, als es meine Aufrichtigkeit und der Prozeß selbst von mir verlangen werden. Denn ich glaubte nicht, jemals eine Fehde mit einer Frau austragen zu müssen - gerade mit einer solchen, die doch alle eher für die Freundin aller als die Feindin von nur irgendeinem hielten. Dennoch will ich sie selbst zuvor fragen, ob sie es lieber habe, daß ich mit ihr in der Strenge und Ernsthaftigkeit früherer Zeiten oder auf die gelassene und milde Weise eines Weltmannes umgehe.)

Nach einer weiteren zweideutig-anzüglichen Schmähung ("amica[] omnium" ist hier nur scheinbar als Kompliment, tatsächlich aber als Vorwurf zu verstehen), ${ }^{50}$ die zudem erneut in der Form eines unvermittelt hinzugefügten Nachtrages erscheint, ${ }^{51}$ gibt Cicero Clodia die Gelegenheit, selbst aussuchen zu können, ob er ihr Verhalten nun aus der Sicht eines altmodischen Tugendwächters ("severe et graviter et prisce") oder aus der eines toleranten Mannes von Welt ("remisse et leniter et urbane") beschreiben soll. In der publizierten Fassung handelt es sich dabei natürlich nur um eine vorgebliche Möglichkeit, Einfluss auf den weiteren Verlauf der Rede zu nehmen, sodass man die Frage zumeist als rein rhetorisch verstanden hat. Wenn man sich das gleiche Angebot aber in der realen Verhandlung - und noch durch eine effektvolle Pause gesteigert - vorstellt, lässt es sich durchaus als weiteres Element der Inszenierung von Spontaneität verstehen. Die konkrete Situation während des Prozesses und damit auch die Wahrscheinlichkeit einer Reaktion Clodias auf eine an sie gerichtete Frage, entzieht sich zwar unserer Kenntnis, aber Catherine Steel hat jüngst mit guten Argumenten dafür plädiert, sich für die römische Gerichtspraxis von der Vorstellung eines die Situation absolut kontrollierenden Redners zu lösen und stattdessen die unvorhersehbaren Elemente stärker in den Blick zu nehmen, die sich aus den Beiträgen der Gegenseite, den Aussagen der Zeugen oder den Reaktionen des Publikums ergeben konn-

$50 \mathrm{Vgl}$. Dyck (2013) Pro Caelio, S. 111: „There is a simple but effective play on several senses of amicus/-a (either 'friend', often with reference to exchange of political and other favors [...] or 'girlfriend, lover' or, especially since it is limited with omnium, 'prostitute'); [...]." Allg. zur Bedeutung von Doppeldeutigkeiten in Ciceros Invektiven Uría (2007) Semantics and Pragmatics, v.a. S. 50-53.

$51 \mathrm{Vgl.}$ Geffken (1973) Comedy, S. 36: "suddenly as in an afterthought". 
ten. ${ }^{52}$ Vor diesem Hintergrund erscheint es nicht unwahrscheinlich, dass antike Leser eine Interaktion mit Clodia zumindest für denkbar hielten und die von Cicero angebotenen Alternativen als ein Element inszenierter Spontaneität wahrgenommen haben.

Was auch immer während der Verhandlung am 4. April 56 v. Chr. passiert ist, Cicero fährt in der publizierten Fassung seiner Rede zunächst einmal mit der ersten von ihm angebotenen Variante fort. Zu diesem Zweck greift er auf ein weiteres dramaturgisches Element zurück und schlüpft in die Rolle eines von Clodias Vorfahren. ${ }^{53}$ Auch hierbei handelt es sich, formal gesehen, um ein gängiges Element der antiken Rhetorik, das unter dem Schlagwort der пробшпопоіа oder der sermocinatio beschrieben und für das sogar genau diese Passage als Paradebeispiel angeführt wird. ${ }^{54}$ Erneut geht Cicero aber über das aus den Schulbüchern zu Erwartende deutlich hinaus, zum einen indem er den Auftritt des von ihm gespielten Ahnen auf der Rednerbühne als eine Totenbeschwörung inszeniert und zum anderen indem er die Wahl des konkreten Vorfahren als spontane Entscheidung präsentiert. ${ }^{55}$ Mit dem bereits 273 v. Chr. verstorbenen Appius Claudius, ${ }^{56}$ der im Alter erblindete und daher das Cognomen Caecus erhalten hatte, lässt er dann - wenig überraschend - aber doch den prominentesten und wegen seiner berühmten Sittenstrenge naheliegendsten Vertreter aus Clodias Familie in Erscheinung treten: ${ }^{57}$

si illo austero more ac modo, aliquis mihi ab inferis excitandus est ex barbatis illis non hac barbula, qua ista delectatur, sed illa horrida, quam in statuis antiquis atque imaginibus videmus, qui obiurget mulierem et pro me loquatur, ne mihi ista forte succenseat. exsistat igitur ex hac ipsa familia aliquis ac potissimum Caecus ille; minimum enim dolorem capiet, qui istam non videbit. qui profecto, si exstiterit, sic aget ac sic loquetur: ,mulier, quid tibi cum Caelio, quid

52 Vgl. Steel (2017) Speech without Limits.

$53 \mathrm{Vgl}$. Cicero, pro Caelio 33-34.

54 Vgl. Quintilian, institutio oratoria 9,2,29-32 (Beschreibung) sowie $3,8,54$ und 12,10,61 (Verweise) mit Lausberg (1960) Handbuch, §§ 820-825.

55 Vgl. hierzu v.a. Klodt (2003) Prozessparteien, S. $88 \mathrm{f}$.

56 Zu dieser bekannten Figur in der historischen Erinnerung Roms vgl. Linke (2000) Caecus.

57 Vgl. Cicero, pro Caelio 33, Übersetzung Fuhrmann (1993). cum homine adulescentulo, quid cum alieno? cur aut tam familiaris huic fuisti, ut aurum commodares, aut tam inimica, ut venenum timeres? non patrem tuum videras, non patruum, non avum, non proavum, non abavum, non atavum audieras consules fuisse? [...].'

(Wenn nun auf jene rauhe Art und Gepflogenheit, dann muß ich jemanden aus der Unterwelt beschwören, und zwar einen von jenen Bärtigen - der nicht ein solches Bärtlein hat, an welchem man heutzutage seine Freude hat, sondern so einen struppigen, wie wir ihn an alten Statuen oder Ahnenmasken sehen -, damit er die Frau rügt und für mich spricht und sie womöglich nicht in Zorn ausbricht gegen mich. Es soll daher einer aus dieser ihrer eigenen Familie auftreten und am ehesten jener berühmte Caecus; denn einer, der sie nicht anblicken wird, wird am wenigsten vom Schmerz mitgerissen werden. Wenn dieser auftritt, wird er bestimmt folgendermaßen mit ihr verfahren und sprechen: „Weib, was hast du mit Caelius, was mit diesem Bürschlein, was mit einem Fremden zu schaffen? Warum bist du mit diesem entweder so vertraut gewesen, daß du ihm Gold überlassen hast, oder so verfeindet, daß du einen Giftanschlag fürchten mußtest? Hattest du nicht gesehen, daß dein Vater, nicht gehört, daß dein Onkel, dein Großvater, dein Urgroßvater, dein Ururgroßvater, dein Urururgroßvater Konsuln gewesen sind? [...].")

In dieser Weise fährt Cicero noch eine Weile fort und hat dabei sicherlich nicht nur inhaltlich und stilistisch einen ,Mann aus der guten, alten Zeit' nachgeahmt, $^{58}$ sondern auch hinsichtlich von Stimme, Mimik und Gestik. ${ }^{59}$ Doch dürfte diese Schauspieleinlage des Redners nicht das einzige gewesen sein, was die Zuhörer an ein Bühnenstück hat denken lassen. Vielmehr drängt sich schon hier der Vergleich zwischen dem altmodischen Appius Claudius und den stereotypen Auftritten

58 Eine genauere stilistische Analyse zeigt allerdings, dass Cicero die Imitation des gehobenen archaischen Stils immer wieder durch die Verwendung umgangssprachlicher Wendungen bricht, was als parodistisches Element aber wieder an die Komödie erinnert. Vgl. Geffken (1973) Comedy, S. $18 f$.

59 Davon ist jedenfalls Quintilian ausgegangen: vgl. institutio oratoria 11,1,39: "utimur enim fictione personarum et velut ore alieno loquimur dandique sunt iis, quibus vocem accomodamus, sui mores; aliter enim P. Clodius, aliter Appius Caecus, aliter Caecilianus ille, aliter Terentianus fingitur." (Wir verwenden nämlich angenommene Rollen, sprechen gleichsam mit der Sprache eines anderen, und dabei müssen wir den Personen, denen wir unsere Sprache leihen, die ihnen eigene Wesensart geben. Anders ist nämlich ein P. Clodius, anders ein Appius Claudius, wieder anders ein Vater bei Caecilius und anders ein Vater bei Terenz zu gestalten.) Übersetzung Rahn (1988). 
der Figur des strengen Vaters in der Komödie auf, in dessen Rolle Cicero dann auch wenig später explizit gegenüber Caelius schlüpft. ${ }^{60}$ Zunächst kommt er aber auf die Alternative zurück, die er Clodia eingangs in Aussicht gestellt hat, und lässt ausgerechnet ihren Bruder P. Clodius als Vertreter der weltmännischen Jugend auftreten, was ihm nicht zuletzt zu weiteren Andeutungen auf einen vermeintlichen Inzest zwischen beiden Gelegenheit gibt. ${ }^{61}$

Damit endet erst einmal der Frontalangriff auf Clodia und Cicero wendet sich anderen Themen zu. In diesem Abschnitt finden sich aber nicht nur eine Reihe hochgradig beleidigender und herabsetzender Äußerungen, sondern auch viele der komödientypischen Elemente, wegen derer die oratio pro Caelio oft als ein Beispiel für eine hybride Gattungskreuzung beschrieben wird. ${ }^{62}$ Diese wiederholten Anleihen beim Theater werden von Cicero als Beitrag zur Unterhaltung des Publikums und als Kompensation für den entgangenen Besuch der parallelen Aufführungen am Fest der Magna Mater eingeführt, sind aber in Wirklichkeit Teil seiner Verteidigungsstrategie und sollen die Anklage als bloße Bühnenintrige entlarven. Zugleich leisten sie aber auch einen wichtigen Beitrag dazu, die gegen die Ankläger allgemein, vor allem aber gegen Clodia direkt gerichteten Schmähungen als spontane Reaktionen des Redners in der Situation des Prozesses erscheinen zu lassen. Dass es sich dabei nur um eine Inszenierung von Improvisationstheater handelt, wird spätestens klar, wenn Cicero die gleichen Strategien auch in der verschriftlichten Version seiner Rede beibehält. Es dürfte sich aber aller Wahrscheinlichkeit nach auch in der realen Situation des Prozesses am 4. April 56 v. Chr. bereits nur um eine Simulation von Spontaneität gehandelt haben, mit der es dem Redner aber gleichwohl gelungen sein dürfte, die Diffamierung seiner Gegner einerseits weniger anstößig erscheinen, andererseits aber - in paradoxer Weise - treffender und damit verletzender wirken zu lassen.

60 Vgl. Cicero, pro Caelio 37-38.

61 Vgl. Cicero, pro Caelio 35-36.

62 Vgl. v.a. Geffken (1973) Comedy; Klodt (2003) Prozessparteien, S. 82-97, und Leigh (2004) Comedy.

\section{Fazit: Invektive Gattungen und die inhärente Innovation}

Obwohl es sich bei Ciceros Verteidigungsrede für Caelius in mancherlei Hinsicht um eine Ausnahme handelt, so kann sie doch verdeutlichen, dass die in der rhetorischen Ausbildung vermittelten Topoi des Tadelns und Schmähens in der Praxis noch einmal gesteigert, aber eben auch in der Art ihrer Präsentation entscheidend variiert wurden. Hierin zeigt sich einerseits die generelle Dynamik von Gattungen, deren Entwicklung sich auch in der Antike weit weniger an die entsprechenden Vorschriften der Poetiken und Rhetorikhandbücher gehalten hat, als häufig angenommen wird: ${ }^{63}$ Zwar galt Homer stets als ideale Verkörperung des Epos, zugleich war es aber nie ein Zeichen gelungener Dichtung, das eigene Werk genau in der Weise zu schreiben, die Homer vorgegeben hatte. Vielmehr erfolgte die imitatio als aemulatio immer mit dem Ziel, die Vorgänger auch zu übertreffen. Außerdem gehörte, vor allem im Rom der Späten Republik und der augusteischen Zeit, also in jener Epoche, die man traditionell als Klassik bezeichnet, das Experiment mit hybriden Formen oder die Gattungskreuzung, wie man im 19. Jahrhundert sagte, ${ }^{64}$ gerade zu den Erkennungszeichen der literarischen Avantgarde. In diese Tendenz eines generic enrichment, um den von Stephen Harrison geprägten Begriff aufzugreifen, ${ }^{65}$ lässt sich auch pro Caelio gut einordnen, sodass zu vermuten steht, dass Cicero sich mit der publizierten Fassung auch als jemand präsentierten möchte, der sich nicht nur auf der Höhe der rhetorischen, sondern auch der allgemein literarischen Entwicklungen seiner Zeit bewegt.

Andererseits gibt es für die Abweichung der individuellen Praxis von einer allen Beteiligten bekannten Theorie aber auch Gründe, die sich aus den spezifischen Rahmenbedingungen für invektives Sprechen oder Schreiben ergeben. Die Triebfeder der Innovationen ist hier nicht in erster Linie die Überbietung der Tradition als solche, sondern das ständige Streben danach, den ste-

63 Vgl. z.B. Farrell (2003) Classical Genre, und Conte/ Most (2012) Genre.

64 Vgl. z.B. Kroll (1924) Studien, 202-224; zum historischen Hintergrund der biologischen Metaphorik Barchiesi (2001) Crossing.

65 Vgl. Harrison (2007) Generic enrichment, v.a. 1-33. 
reotypen Erwartungen auszuweichen und unter anderem mit Hilfe scheinbarer Spontaneität oder artifizieller Mündlichkeit den Anschein zu erwecken, dass die jeweiligen Beleidigungen - ganz oder zumindest teilweise - unvorbereitet aus der Situation heraus entstanden sind, weil sie dann sowohl als weniger problematisch wahrgenommen werden als auch zugleich eine stärkere Wirkung entfalten. Bei der Simulation spontaner Mündlichkeit handelt es sich aber letztlich nur um einen - wenn auch wichtigen - Teilbereich eines größeren Phänomens. An den Spannungen zwischen der ars invectiva, wie sie in den Lehrbüchern vermittelt wird, und der Praxis der Rede, wie wir sie in Rom vor allem bei Cicero greifen können, zeigt sich nicht zuletzt, dass invektiven Gattungen eine gleichsam institutionalisierte Innovation in besonderem Maße inhärent ist.

\section{Literaturverzeichnis}

\section{Quellen}

Achard, Guy (21997): Rhétorique à Herennius. Paris: Les belles lettres.

Austin, R.G. ( ${ }^{31960): ~ M . ~ T u l l i ~ C i c e r o n i s ~ p r o ~ M . ~ C a e l i o ~}$ oratio. Oxford: Oxford University Press.

Adamietz, Joachim (1966): M. F. Quintiliani institutionis oratoriae liber III mit einem Kommentar. München: Fink.

Diels, Hermann/Kranz, Walther (61952): Die Fragmente der Vorsokratiker, griech.-dt., 2. Bde. Berlin: Weidmannsche.

Dyck, Andrew R. (2013): Cicero: Pro Marco Caelio. Cambridge: Cambridge University Press.

Fuhrmann, Manfred (1993): Cicero: Die politischen Reden, Bd. 2, lat.-dt. München: Artemis \& Winkler.

Fuhrmann, Manfred (1995): Marcus Tullius Cicero, Die Reden gegen Verres - in C. Verrem, lat. - dt., Bd. 1. Zürich: Artemis \& Winkler.

Hubert, Curt (1938): Plutarchi Moralia, vol. IV [enthält u.a.: Quaestiones convivales]. Leipzig: Teubner.

Kassel, Rudolf (1976): Aristotelis Ars rhetorica. Berlin: De Gruyter.

Kennedy, George A. (2003): Progymnasmata. Greek textbooks of prose composition and rhetoric. Leiden: Brill 2003.

Kumaniecki, Kazimierz F. (1995): M. Tulli Ciceronis scripta quae mansuerunt omnia Fasc. 3 de oratore. Stuttgart/Leipzig: Teubner.

Maslowski, Tadeusz (1995): M. Tulli Ciceronis scripta quae mansuerunt omnia Fasc. 23: Orationes in P. Vatinium testem; pro M. Caelio. Stuttgart/Leipzig: Teubner.
Novokhatko, Anna A. (2009): The Invectives of Sallust and Cicero. Critical Edition with Introduction, Translation, and Commentary. Berlin: De Gruyter.

Nüßlein, Theodor (1994): Rhetorica ad Herennium, lat.-dt. Düsseldorf: Artemis \& Winkler.

Nüßlein, Theodor (1998): Cicero: De inventione; De optimo genere oratorum; lat.-dt. Düsseldorf: Artemis \& Winkler.

Nüßlein, Theodor (2007): Cicoer: de oratore, lat.-dt. Düsseldorf: Artemis \& Winkler.

Patillon, Michel (2008-2014): Corpus rhetoricum, 5 Bde. Paris: Les belles lettres.

Peterson, William (1907): M. Tulli Ciceronis Orationes, vol. 3: Divinatio in Q. Caecilium, in C. Verrem. Oxford: Oxford University Press.

Rahn, Helmut (1988) [ $\left.{ }^{6} 2015\right]$ : Marcus Fabius Quintilianus: Ausbildung des Redners. Zwölf Bücher, lat.-dt. . Darmstadt: WBG.

Rapp, Christof (2002): Aristoteles: Rhetorik. Übersetzt und erläutert. Berlin: Akademie Verlag.

Reynolds, L.D. (1965): L. Annaei Senecae ad Lucilium epistulae morales, 2. Bde. Oxford: Oxford University Press.

Schönberger, Otto/Schönberger, Eva (2019): Aphthonios von Antiochia: Progymnasmata / Vorübungen. Griechischer Text, Nachwort, deutsche Übersetzung, Einführung und Anmerkungen. Würzburg: Königshausen u. Neumann.

Stroebel, Eduard (1965) [ $={ }^{11915]: ~ M . ~ T u l l i ~ C i c e o r n i s ~}$ scripta quae mansuerunt omnia Fasc. 2: Rhetorici libri duo qui vocantur de inventione. Stuttgart: Teubner.

Winterbottom, Michael (1970): M. Fabi Quintiliani institutionis oratoriae libri XII. Oxford: Oxford University Press.

\section{Forschungsliteratur}

Adamietz, Joachim (1960): Ciceros de inventione und die Rhetorik ad Herennium. Marburg: Diss.

Andersen, Øivind (1996): Lingua suspecta on concealing and displaying the art of rhetoric. In: Symbolae Osloenses 71, S. 68-86.

Arcellaschi, André (1997): Le Pro Caelio et le théâtre. In: Revue des études Latines 75, S. 164-172.

Arena, Valentina (2007): Roman Oratorical Invective. In: Dominik, William/Hall, Jon (Hgg.): A Companion to Roman Rhetoric. Malden: Wiley-Blackwell, S. $149-160$.

Bablitz, Leanne (2007): Actors and Audience in the Roman Courtroom. London: Routledge.

Barchiesi, Alessandro (2001): The Crossing. In: Harrison, Stephen J. (Hg.): Texts, Ideas, and the Classics: Scholarship, Theory, and Classical Literature. Oxford: Oxford University Press, S. 142-163.

Beard, Mary (2014): Laughter in ancient Rome: on joking, tickling, and cracking up. Berkeley: University of California Press. (dt.: Das Lachen im alten Rom: eine Kulturgeschichte. Darmstadt: WBG 2016). 
Berardi, Francesco (2017): La retorica degli esercizi preparatori: glossario ragionato dei Progymnásmata. Hildesheim: Georg Olms Verlag.

Blänsdorf, Jürgen (2001): Cicero auf dem Forum und im Senat: Zur Mündlichkeit der Reden Ciceros. In: Benz, Lore (Hg.): Die römische Literatur zwischen Mündlichkeit und Schriftlichkeit. Tübingen: Narr Francke Attempto, S. 205-228.

Chiron, Pierre (2011): Relative Dating of the Rhetoric to Alexander and Aristotle's 'Rhetoric'. In: Rhetorica 29, S. 236-262.

Conte, Gian Biagio/Most, Glen W. (2012): Genre. In: The Oxford Classical Dictionary. $4^{\text {th }}$ Edition. Oxford: Oxford University Press, S. 609-610.

Craig, Christopher (2004): Audience Expectations, Invective, and Proof. In: Powell, Jonathan/Patterson, Jeremy (Hgg.): Cicero the Advocate. Oxford: Oxford University Press, S. 187-213.

Fantham, Elaine (2004): The Roman World of Cicero's De Oratore. Oxford: Oxford University Press.

Farrell, Joseph (2003): Classical Genre in Theory and Practice. In: New Literary History 34, S. 383-408.

Fuhrmann, Manfred (1965): Untersuchungen zur Textgeschichte der pseudo-aristotelischen Alexander-Rhetorik (der T'́x $\mathbf{x} \eta$ des Anaximenes von Lampsakos). Mainz: Akademie der Wissenschaften.

Geffcken, Katherine A. (1973) [21995]: Comedy in the Pro Caelio. With an Appendix on the In Clodium et Curionem. Leiden: Brill.

Günther, Rosmarie (2000): Sexuelle Diffamierung und politische Intrigen in der Republik. P. Clodius Pulcher und Clodia. In: Späth, Thomas/Wagner-Hasel, Beate (Hgg.): Frauenwelten in der Antike. Geschlechterordnung und weibliche Lebenspraxis. Stuttgart: J.B. Metzler, S. 227-241.

Hall, Jon (2014): Cicero's Use of Judicial Theater. Ann Arbor: University of Michigan Press.

Harders, Ann-Cathrin (2008): Suavissima Soror. Untersuchungen zu den Bruder-Schwester-Beziehungen in der römischen Republik. München: C.H. Beck.

Hartmann, Elke (2007): Frauen in der Antike. München: C.H. Beck.

Harrison, Stephen J. (2007): Generic enrichment in Vergil and Horace. Oxford: Oxford University Press.

Hesk, Jon (1999): The Rhetoric of Anti-rhetoric in Athenian Oratory. In: Goldhill, Simon/Osborne, Robin (Hgg.): Performance Culture and Athenian Democracy. Cambridge: Cambridge University Press, S. 201-230.

Hickson-Hahn, Frances (1998): What's so Funny? Laughter and Incest in Invective Humor. In: Syllecta Classica 9, S. 1-36.

Humbert, Jules (1925): Les plaidoyers écrits et les plaidoiries réelles de Cicéron. Paris: Les Presses Universitaires de France.

Keeline, Thomas J. (2018): The Reception of Cicero in the Early Roman Empire: The Rhetorical Schoolroom and the Creation of a Cultural Legend. Cambridge: Cambridge University Press.
Klodt, Claudia (2003): Prozessparteien und politische Gegner als dramatis personae. Charakterstilisierung in Ciceros Reden. In: Schröder, Bianca-Jeanette/ Schröder, Jens Peter (Hgg.): Studium declamatorium: Untersuchungen zu Schulübungen und Prunkreden von der Antike bis zur Neuzeit. Leipzig: K. G. Saur, S. 35-106.

Koster, Severin (1980): Die Invektive in der griechischen und römischen Literatur. Meisenheim am Glan: Anton Hain.

Kroll, Wilhelm (1924): Studien zum Verständnis der römischen Literatur. Stuttgart: J. B. Metzler.

La Bua, Giuseppe (2019): Cicero and Roman education: the reception of the speeches and ancient scholarship. Cambridge: Cambridge University Press.

Langlands, Rebecca (2018): Exemplary ethics in ancient Rome. Cambridge: Cambridge University Press.

Linke, Bernhard (2000): Appius Claudius Caecus - ein Leben im Zeitalter des Umbruchs. In: Hölkeskamp, Karl-Joachim/Stein-Hölkeskamp, Elke (Hgg.): Von Romulus zu Augustus. Große Gestalten der römischen Republik. München: C.H. Beck, S. 69-78.

Lausberg, Heinrich (1960): Handbuch der literarischen Rhetorik. Eine Grundlegung der Literaturwissenschaft, 2 Bde. München: Max Hueber.

Leigh, Matthew (2004): The Pro Caelio and Comedy. In: Classical Philology 99, S. 300-335.

May, James M. (1988): Trials of Character: The Eloquence of Ciceronian Ethos. Chapel Hill: University of North Carolina Press.

Papaioannou, Sophia/Serafim, Andreas/da Vela, Beatrice (Hgg.) (2017): The Theatre of Justice. Aspects of Performance in Greco-Roman Oratory and Rhetoric. Leiden: Brill.

Pausch, Dennis (im Erscheinen): Comic Invective in Cicero's speech pro M. Caelio. In: Papaioannou, Sophia/Serafim, Andreas (Hgg.): Comic invective in ancient Greek and Roman oratory. Berlin: De Gruyter.

Piepenbrink, Karen (2020): Die Rhetorik des Aristoteles und ihr Verhältnis zum historischen Kontext. Stuttgart: Franz Steiner.

Powell, Jonathan (2007): Invective and the orator: Ciceronian theory and practice. In: Booth, Joan ( $\mathrm{Hg}$.$) : Cicero on the Attack: Invective and subversion$ in the orations and beyond. Swansea: The Classical Press of Wales, S. 1-24.

Riggsby, Andrew (1999): Crime and Community in Ciceronian Rome. Austin: University of Texas Press.

Roller, Matthew B. (2018): Models from the past in Roman culture: a world of exempla. Cambridge: Cambridge University Press.

Schloemann, Johann (2019): ,I have a dream' Die Kunst der freien Rede von Cicero bis Barack Obama. München: C.H. Beck.

Skinner, Marilyn B. (2011): Clodia Metelli. The Tribune's Sister. Oxford: Oxford University Press.

Steel, Catherine (2013): Cicero, Oratory and Public Life. In: Dies. (Hg.): The Cambridge Companion to Cicero. Cambridge: Cambridge University Press, S. 160-170. 
Steel, Catherine (2017): Speech without Limits: Defining Informality in Republican Oratory. In: Papaioannou, Sophia/Serafim, Andreas/da Vela, Beatrice (Hgg.): The Theatre of Justice. Aspects of Performance in Greco-Roman Oratory and Rhetoric. Leiden: Brill, S. $75-89$.

Stroh, Wilfried (1975): Taxis und Taktik. Ciceros Gerichtsreden. Stuttgart: Teubner.

Stroh, Wilfried (2009): Die Macht der Rede. Eine kleine Geschichte der Rhetorik im alten Griechenland und Rom. Berlin: Ullstein.

Tatum, W. Jeffrey (2007): Invective Identities in Pro Caelio. In: Smith, Christopher/Covino, Christopher (Hgg.): Praise and Blame in Roman Republican Rhetoric. Swansea: The Classical Press of Wales, S. $165-179$.

Tempest, Kathryn L. (2013): Staging a Prosecution: Aspects of Performance in Cicero's Verrines. In: Kremmydas, Christos/Powell, Jonathan/Rubinstein, Lene (Hgg.): Profession and Performance: Aspects of Oratory in the Greco-Roman World. London: Institute of Classical Studies, School of Advanced Study, University of London, S. 41-72.
Thurn, Anabelle (2018): Rufmord in der späten römischen Republik. Charakterbezogene Diffamierungsstrategien in Ciceros Reden und Briefen. Berlin: De Gruyter.

Till, Dietmar (2009): Verbergen der Kunst (lat. dissimulatio artis). In: Ueding, Gerd (Hg.): Historisches Wörterbuch der Rhetorik, Bd. 9: St-Z. Tübingen: Niemeyer, Sp. 1034-1042.

Uría, Javier (2007): The semantics and pragmatics of Ciceronian invective. In: Booth, Joan ( $\mathrm{Hg}$.$) : Cicero on$ the attack. Invective and subversion in the orations and beyond. Swansea: The Classical Press of Wales, S. 47-70.

Volpe, Michael (1977): The Persuasive Force of Humor: Cicero's Defense of Caelius. In: Quarterly Journal of Speech 63, S. 311-323.

Williams, Hamisch (2007): Cicero: pro Caelio: What was it that most undermined Clodias' case - her character, the prejudices of Roman men, the Skills of Cicero or ...? In: Akoterion 52, S. 121-127.

Zillinger, Wilhelm (1911): Cicero und die altrömischen Dichter. Würzburg: Franz Staudenraus. 